\title{
TRANSICIÓN Y CONSTRUCCIÓN DE LA PATERNIDAD
}

\section{TRANSITION AND CONSTRUCTION OF FATHERHOOD}

\author{
LILIANA NIERI* \\ *Doctora en Psicología, especialista en Psicología Perinatal. Becaria Posdoctoral del Consejo Nacional de \\ Investigaciones Científicas y Técnicas (CONICET) y Docente de la Fundación Universidad Argentina de la \\ Empresa (UADE). E-Mail: liliananieri@hotmail.com \\ Consejo Nacional de Investigaciones Científicas y Técnicas (CONICET) \\ Fundación Universidad Argentina de la Empresa (UADE) \\ Ciudad Autónoma de Buenos Aires - República Argentina
}

\section{RESUMEN}

Algunos hechos históricos trajeron aparejadas consecuencias sociales y psicológicas que llevaron a replantear las funciones de los padres. Esto llevó a que las investigaciones centraran su interés en estudiar qué le ocurría al padre ante su paternidad. Por lo tanto el trabajo que se informa tuvo como objetivo realizar una revisión bibliográfica relacionada con la transición de la paternidad. En el mismo se pueden evidenciar diversas investigaciones que dan cuenta de distintos procesos emocionales que atraviesa el padre y cómo estos influyen en la relación con su familia de origen, pareja e hijo. Se observó que factores tales como la relación matrimonial, las demandas de trabajo, las relaciones tempranas con su familia de origen influyen en la construcción de la identidad paterna. Los estudios revisados destacan la importancia de entender qué le ocurre al padre durante ese período, ya que como lo viva incidirá en la relación con su hijo.

Palabras clave: Paternidad; Transición; Construcción; Identidad; Relación; Embarazo; Nacimiento.

\section{ABSTRACT}

For decades, the researchers agreed that the father's role was just being an economic provider and he was considered secondary in the function of parenting. Thus, most of studies focused in the mother's psychological processes through the pregnancy, childbirth and postpartum, letting of father's role side out. However, according with historical changes as French revolution, industrial revolution and the both world wars, the psychologists throw their interest into know which the father role is and how he influences in the psychological development of their children. One of the first psychologists to study the role of the father was S. Freud, he thinks that father's role was to distance the mom to their son; the father represents authority, rules, law, order, the opening to the world. In this theory, the interaction between father and son begin around 2 years of the child. Later this theory was refused for different psychologist who found that the father's early involvement has a positive impact on the development of their children. From the 70's the interest was to know how is the man's psychological change that became a father, and how the transition to the construction of parenthood is. As the research progressed, the researchers discovered that the parents during the pregnancy of his wife manifested the need to feel included in this process. So, the psychologists began to ask questions about what happen to the father in childbirth? Greenberg and Morris (1974) think that the childbirth is the most intensive moment in an emotional level to build the parenthood, so they start to study the emotions and 
concluded that there is a stage called: engrossment, it is about a father's innate potential and it develop with the childbirth. Based on these theories the aim of this research is make a bibliographic review to better understand the father's transition, including researches that explained different father's emotional processes and how this emotional processes influence in the relationship with his family of origin, his couple and his sons. The method used was a bibliographic search in the databases: Pub Med, Redalyc, Scielo, MEDLINE, EBSCO, and APA, from 1970 to date. We found 150 articles and books. After a first reading 63 articles were not included because it did not meet with the criteria. Finally, we included 89 articles and books for this review. This studies shows that the transition to paternity is a multidimensional process in terms of individual, partner, work, family of origin, among others. This means that the father goes through a process of transition, in which: his history as a child, the relationship with his partner and his current situation play an important role in the development of his paternal identity. Researches highlights the importance of understanding what happens to the father during his transition, as the way he crosses it affects the relationship with his son or daughter. Thus the fatherhood is not just a biological act, but there are some factors that join in for construction and organize the fatherhood (Palkovitz \& Palm, 2009). Like the mother, a father has a vital and evolutionary crisis; in this period interact his personal history, personality, currently situation, relationships and baby's characteristics (Nieri, 2015; Oiberman, 2008). Know these studies allow understand and explains the father's affective process through the fatherhood, and how the father develops a meaning about his new state is and what the consequences are. Also, previous bibliography shows evidence to the necessity to understand what is happening and how this influence in his relationship with his wife and baby (Habib \& Lancaster, 2010; Moreno-Rosset et al., 2016).

Key words: Transition; Parenthood; Construction; Identity; Relationship; Pregnancy; Birth.

\section{INTRODUCCIÓN}

Durante años las investigaciones se han centrado en el estudio de la maternidad en re- lación con el embarazo, parto y puerperio. Estas concluían que la madre era la principal cuidadora y sostén de su hijo. El conocer y entender lo que le ocurría a la madre durante dicho período era central para el futuro del niño. Desde el punto de vista social, la función del padre consistía en proveer el soporte económico de su familia y así poder ayudar a la madre a que el embarazo llegue a término. Las primeras investigaciones psicológicas concluyeron que el padre atravesaba por un proceso de transición, en donde su historia como hijo, la relación con su pareja y su situación actual, configuraban la manera en que se relacionaba con su hijo (Oiberman, 2008). En un comienzo se creía que dicha transición se generaba ante el nacimiento del hijo (Bouchard, 2014; Greenberg \& Morris, 1974); sin embargo, a medida que las investigaciones avanzaron sobre ese tema, descubrieron que los padres durante el embarazo de su esposa, manifestaban la necesidad de sentirse incluidos en dicho proceso (Bouchard, 2014). Es así que se comienza a pensar en qué le ocurría al padre durante la gestación de su hijo.

A partir de esto, muchas investigaciones centraron su interés en estudiar el proceso de transición hacia la paternidad durante la gestación de su hijo (Draper, 2003; Habib \& Lancaster, 2010; Kowlessar, Fox \& Wittkowski, 2014; May, 1982).

El nacimiento de un bebé trae consigo cambios importantes en la vida de los nuevos padres. Esta transición a la paternidad significa el establecimiento de nuevos lazos emocionales y la entrada en una nueva vida (Feeney, Hohaus, Noller \& Alexander, 2001; Kowlessar, Fox \& Wittkowski, 2014; Moreno-Rosset, Arnal-Remón, Antequera-Jurado \& Ramírez-Uclés, 2016). Para Casullo

“el tránsito a la paternidad implica la adquisición de ciertas habilidades, cambios en la identidad así como la construcción de significados" (2004, p. 56).

Dentro de este proceso hay tres áreas de dificultad para los hombres durante el período prenatal. La primera son los sentimientos 
afectados de no realidad, que provienen de la carencia de pruebas tangibles de la existencia de su hijo aún no nacido y el deseo de desarrollar un enlace emocional con el niño (Gage \& Kirk, 2002).

La segunda área problemática para los padres pertenece a la relación de la pareja, es decir, un desequilibrio percibido en la relación con su compañero, implicando una divergencia entre expectativas masculinas y femeninas sobre la relación y las necesidades (Kowlessar et al., 2014).

Finalmente, la tercera dificultad se relaciona con la formación de la identidad paternal, la cual requiere un cambio de identidad principal del papel a desarrollar (Habib \& Lancaster, 2010; Strauss \& Goldberg, 1999).

En relación a lo anterior, McCall y Simmons (1978) sostienen que el contenido de identidad es teóricamente significativo, ya que la conducta del rol relacionado con los hombres se ve reflejada en los diversos significados de quienes son padres. Lo que significa que no hay una sola manera de ser padre, y por lo tanto hay variaciones en el contenido del rol e identidad del padre (Höfner, Schadler \& Richter, 2011).

Para Durkheim (1951) y Cast (2004) la transición a la paternidad produce sentimientos tanto positivos como negativos que inciden en la relación con su hijo (Fillo, Simpson, Rholes \& Kohn, 2015; Höfner et al., 2011). Por ejemplo, el bebé puede dar a los nuevos padres una mayor satisfacción con la vida, pero al mismo tiempo, el cambio a una tríada puede generar presión social en relación con el impacto de convertirse en padre (Keizer, Dykstra \& Poortman, 2010).

En virtud de lo expuesto, este trabajo tuvo como objetivo realizar una revisión bibliográfica acerca de la transición a la paternidad. Se evidencian diversos trabajos acerca de los distintos procesos emocionales y psicológicos que atraviesa el padre y cómo estos influyen en la relación con su familia de origen, pareja e hijo por nacer y / o nacido (Moreno-Rosset et al., 2016).

También es importante entender cómo es la transición hacia la paternidad, la conformación de la identidad paterna y las relacio- nes que van a influir en el nivel de participación e involucramiento del padre con su hijo por nacer y / o nacido.

\section{MÉTOdO}

\section{BÚSQUEDA BIBLIOGRÁFICA}

Se realizó una búsqueda bibliográfica a partir del año 1970 hasta la fecha, en las siguientes bases de datos: PubMed, Redalyc, SciELO, MEDLINE, EBSCO y APA.

Los términos de búsqueda utilizados fueron: paternidad, relación de pareja, identidad paterna, transición a la paternidad, nacimiento de un hijo, vínculo padre - hijo, embarazo y experiencia familiar. Las seis bases de datos dieron como resultado un total de 150 artículos y libros.

También se realizó una búsqueda no sistemática de la literatura basada en publicaciones de reconocidos autores dentro de los campos de la paternidad y arrojó 45 estudios diferentes, que parecían relevantes según el título y el resumen.

\section{CRITERIOS DE INCLUSIÓN}

Se consideraron todos los estudios que cumplían los siguientes criterios de inclusión: (1) Artículos referidos exclusivamente a la transición e identidad paterna. (2) Artículos relacionados con la transición e identidad paterna y el nacimiento de un hijo sano. (3) Que no fueran artículos históricos sobre la función y rol del padre y (4) que los artículos fueran Fulltext.

\section{CRITERIO de EXCLUSIÓN}

De los 150 artículos y libros seleccionados, 63 no cumplían con los criterios de inclusión establecidos, ya que los mismos se referían a la transición materna, a la transición e identidad paterna ante el nacimiento de un hijo con patologías genéticas y / o no eran Fulltext. Finalmente, se incluyeron 89 artículos y libros. 


\section{Resultados}

\section{TRANSICIÓN A LA PATERNIDAD}

En la década de los años 80, la mayoría de las investigaciones centraron su interés en comprender la formación temprana de la familia y el proceso de transición a la paternidad. Sin embargo, las primeras investigaciones tomaron dicha situación como una crisis; de esta manera, la mayoría de los estudios se han interesado en conocer cómo la transición hacia la paternidad influye en la relación de pareja. Estos primeros intentos de analizar empíricamente los efectos de dicha transición en las relaciones maritales parten de la hipótesis de que el nacimiento de un hijo genera una crisis en las parejas. Así, Hill (1949) define a esta crisis como una situación que se genera después de un evento importante. Hay tres factores que determinan si se ha producido una crisis: definición de caso, dificultades asociadas con el evento y la disponibilidad de los recursos familiares para responder a dicho evento (LeMasters, 1957).

Dentro de esta misma línea, Bibring (1959) postulaba que la transición a la paternidad está marcada por momentos decisivos y específicos en la vida del individuo, que da un paso hacia las nuevas funciones, es decir, que es un fenómeno para el desarrollo, es un punto sin retorno entre una fase y la siguiente.

No obstante, Rossi (1968) cambia el enfoque conceptual de crisis, entendiendo que la transición implica un cambio normativo de una etapa a otra. Esto significa que las transiciones son períodos de cambio (Price, Mc Kenry \& Murphy, 2000), es decir, que el embarazo y la transición a la paternidad marcan un período de desarrollo importante para los padres, por la relación cuidador - niño y el desarrollo del bebé.

Nyström y Öhrling (2004) informan que algunos padres fueron dominados por sentimientos de amor y alegría inspirados por la nueva familia. Dicha satisfacción y confianza se relacionaron con sentimientos de compartir las preocupaciones con su compañero.

Las investigaciones han demostrado consistentemente que el nacimiento de un niño es a menudo un evento estresante y lleva consigo cambios más profundos que cualquier otro estado en el desarrollo durante el ciclo de vida (Ohashi \& Asano, 2012).

De igual manera, dichas transiciones dan lugar a una reorganización de los roles y de las relaciones con otras personas significativas (Bouchard, 2012). Por ejemplo, en el ámbito de la paternidad, la transición para algunos padres puede extenderse durante varios años, mientras que para otros puede terminar poco después del nacimiento del niño (Reynolds, Houlston \& Coleman, 2014). Así la transición genera nuevos significados que comprenden las expectativas, los niveles de habilidad y conocimiento, el ambiente alrededor de los padres y el bienestar emocional y físico (Ohashi \& Asano, 2012; Schumacher \& Meleis, 1994).

Esto quiere decir que en el curso de la generación de una nueva vida, todos los padres se ven sometidos a una transformación del sí mismo a nivel emocional y psicológico (KatzWise, Priess \& Hyde, 2010). Esto significa que la transición es un proceso gradual, físico, psicológico y espiritual que consiste en el movimiento de la vida normal, por un período de reorganización psicológica y adaptación, a la incorporación de las nuevas circunstancias (Swain, 2011; Genesoni \& Tallandini, 2009).

Schumacher y Meleis (1994) sostienen que la teoría de la transición sugiere que este proceso se ve influenciado por las creencias culturales y las actitudes de las personas $(\mathrm{Ge}-$ nesoni \& Tallandini, 2009).

Para Kowlessar y colaboradores (2014) la transición a la paternidad comienza con la confirmación del embarazo y continúa hasta los primeros meses después del nacimiento de un niño, en donde los roles (por ejemplo, proveedor, cónyuge) se vuelven particularmente relevantes.

En consecuencia, es un momento en que los sentimientos de éxito y la competencia en estas funciones pueden dar lugar a un ajuste saludable a la paternidad, mientras que los sentimientos de fracaso e incompetencia en estos roles pueden conducir a una mala adaptación a la paternidad (Cowan, P.A. \& Cowan, C.P., 1988; Moreno-Rosset et al., 2016). 
Los procesos psicológicos de la transición se pueden ver reflejados en tres períodos de tiempo, el período prenatal que consiste en una fase de reorganización basada en la aparición del estado de la paternidad y dicho proceso requiere la integración gradual de los papeles del padre (Bouchard, 2014). En relación al segundo período, los estudios que han investigado la paternidad durante el nacimiento sostienen que es el período más intenso a nivel emocional, de la transición a la paternidad (Habib \& Lancaster, 2010). Finalmente, durante la etapa postnatal, los hombres se encuentran esforzándose por equilibrar las necesidades personales y de trabajo con las nuevas demandas del estado de padre y su nueva autoimagen. No obstante, un elemento común en los tres períodos es la importancia de la calidad de la relación del hombre con su pareja (Draper, 2003; Miller, 2011; MorenoRosset et al., 2016).

Dentro de esta línea, Kowlessar y colaboradores (2014) sostienen que el proceso de transición a la parentalidad (el cual comienza durante el embarazo y los primeros meses de vida de un hijo) es el reconocimiento del niño como tal, lo que induce al desarrollo del sentimiento de ser padre que es el reconocimiento de la dependencia y de la imposibilidad del niño de satisfacer sus propias necesidades. Asimismo, se ha demostrado que esta transición no solo concierne a la mujer, sino que incluye al padre, es decir que el nacimiento de un niño es un importante período de transición para las familias y lleva a la pareja a hacer adaptaciones significativas (Cox, Paley, Burchinal \& Payne, 1999). Esta transición puede ser muy estresante para muchos padres, debido a que el nacimiento de un niño aumenta exponencialmente la carga de trabajo familiar, lo que requiere una negociación y coordinación de tareas (Deave \& Johnson, 2008).

Según P.A Cowan y C.P. Cowan (1988), cuando los varones llegan a ser padres ocurren tres cambios: incorporan la paternidad a su identidad, tienen la sensación de que han perdido el control de sus vidas y sus aspiraciones personales tienden a desarrollarse exponencialmente (Palkovitz \& Palm, 2009).
Tal como sostienen Herzog y Lebovici (1995) el concepto de paternidad no significa ser padre en el sentido biológico del término, sino también poder imaginarse su descendencia.

Esto significa que la paternidad no solo representa un desarrollo en el adulto y un movimiento para una nueva posición social, sino que implica un

"proceso psicoafectivo por el cual un hombre realiza una serie de actividades en lo concerniente a concebir, proteger, aprovisionar y criar a cada uno de sus hijos jugando un importante y único rol en el desarrollo del mismo, distinto al de la madre" (Oiberman, 2008, p. 104).

Dicho proceso es vivido por el hombre en función de su historia personal, su actualidad y las vivencias en relación a su hijo por nacer $\mathrm{y} / \mathrm{o}$ recién nacido. La forma en que se unan dichos acontecimientos se verá reflejada en las conductas que asuma en relación a su pareja e hijo (Nieri, 2015; Ohashi \& Asano, 2012; Oiberman, 2008).

Así el futuro padre cursa un proceso de transición hacia la paternidad, en tanto la misma provoca cambios en el estado de salud, en las relaciones, en las expectativas y / o habilidades y en la identidad personal y social (Schumacher \& Meleis, 1994; Testa, 2010).

Para C.P. Cowan y P.A. Cowan (2000) los padres transitan diversas etapas, la primera es su ansiedad acerca de convertirse en padre (la vida interior), luego surge la calidad de las relaciones en la familia, seguida por las exigencias de un trabajo fuera del hogar (estrés fuera de la familia) y por último, la negociación de nuevos roles y decisiones dentro de la familia -la calidad del matrimonio- (Testa, 2010).

En resumen, diversas investigaciones concluyeron que la mayoría de los padres relatan cambios en la naturaleza e intensidad de sus sentimientos (Cowan, P.A. \& Cowan, C.P., 1988; Habib \& Lancaster, 2010; Strauss \& Goldberg, 1999) y amor propio (Berman \& Pedersen, 1987). Los sentimientos pueden 
ser negativos o positivos. Los primeros están caracterizados por ansiedad, ambivalencia $u$ hostilidad, confusión, distanciamiento (Barclay, Donovan \& Genovese, 1995; Buist, Morse \& Durkin, 2003; Rholes et al., 2011; Kowlessar et al., 2014) y síntomas depresivos (Arnal-Remón, Moreno-Rosset, RamírezUclés \& Antequera-Jurado, 2015). Sin embargo, los sentimientos positivos están caracterizados por ser afecto, realización, maravilla y regocijo (Greenberg \& Morris, 1974; Lupton \& Barclay, 1997; Testa, 2010).

\section{IDENTIDAD PATERNA}

Para entender qué ocurre con los cambios en la paternidad, es necesario centrarse en la definición de identidad, la misma postula que la conducta de una persona se delimita en función de la posición que ocupa en la sociedad (Kuhn, 1960). Tal como se aplican en las investigaciones sobre paternidad, las teorías proponen que el elemento clave de la participación del padre es el grado en que un padre se identifica con la situación y los roles asociados con ser padre. Además, postulan que estas autopercepciones se organizan jerárquicamente, haciendo que en un momento del proceso, algunos roles del padre sean más importantes que otros (Ihinger-Tallman, Pasley \& Buehler, 1993).

Estas diversas identidades reflejan la integración de los individuos a nivel social, las expectativas con sus propias creencias y las expectativas en cuanto a lo que significa ocupar cierto papel -abastecedor, proveedor- y que dichas identidades posteriormente dirigen el comportamiento (Burke \& Reitzes, 1991).

Asimismo, las teorías sostienen que la identidad está formada por modelos multidimensionales, lo que significa que no se limita solo a la identidad individual, ya que tanto los aspectos culturales como los sociales van a influir en la manera de como un padre ejerza su función (de Montigny, P. \& de Montigny, F., 2013). Por otra parte, está íntimamente vinculada con sus relaciones sociales del pasado y del presente, y por lo tanto incorpora varios elementos de los modelos de imagen, como la relación conyugal y la historia evolutiva (Belsky \& Kelly, 1994; Bouchard, 2012; Cowan, C.P. \& Cowan, P.A., 2000; Testa, 2010). Esto significa que la transición a la paternidad anuncia un proceso de transmisión intergeneracional en donde la experiencia de cómo fue criado actúa como la fuente inicial de la formación o constitución mental acerca del rol de ser padre, a pesar de que puede permanecer latente o encubierta hasta que el disparador de la realidad, convertirse en padre, evoca las representaciones mentales de las experiencias anteriores de la paternidad (Bouchard, 2012; Habib, 2012).

También Pleck (1997) sostiene que la identidad paternal puede ser vista como una integración de la historia, las características de la personalidad en el desarrollo de la persona y las creencias relacionadas con la paternidad (de Montigny, P. \& de Montigny, F., 2013).

La investigación sugiere que la percepción del hombre de sí mismo en el estado del padre, influye en la relación con sus hijos. Por ejemplo, la teoría de la identidad se ha utilizado para explicar la participación paterna en relación con la función del padre y la relación de pareja (Bouchard, 2012; Palkovitz \& Palm, 2009).

En términos de esta teoría, existe un importante factor que determina la conducta paterna, la misma se basa en la importancia de la condición de padre de un hombre, es decir, el conjunto jerárquico de otros estados (por ejemplo, trabajador, hijo, amigo, etc.)

Asimismo, es necesario considerar que el papel de la maternidad es visto por la sociedad como elemento central de la identidad de una mujer, en el que los hombres tienden a percibir la paternidad como algo que hacen, mientras que las mujeres suelen experimentar la maternidad como algo que son (Ehrensaft, 1987). Las investigaciones previas sugieren que los padres son vistos por las madres como una ayuda en lugar de compartir la responsabilidad parental (Cowan, C.P. \& Cowan, P.A., 2000).

Sin embargo, para entender cómo se desarrolla la identidad paterna y cuáles son los factores que inciden en la misma durante la 
transición, es necesario tomar dos perspectivas teóricas que explican, desde diferentes miradas, el desarrollo de identidad paterna (Palkovitz \& Palm, 2009). Dichas teorías seleccionadas hacen hincapié en el desarrollo de la identidad como una integración del individuo, la familia, la cultura y el ambiente social.

\section{Modelo de la IDENTIDAd de Rol de McCall y SIMMONS (1978)}

Esta teoría pone el énfasis en la importancia de la negociación y permite observar formas de interacción particulares entre las personas, así como intercambios a lo interno, entre quienes han asumido un rol. De acuerdo con esta teoría, el sujeto adopta un rol, con las implicaciones que éste tiene: significados, expectativas que se tienen de él, así como de la negociación que cada ocupante pueda hacer (Bouchard, 2012).

Esta identidad de rol se deriva de la visión imaginaria que una persona tiene de sí misma. El rol de las identidades constituye la fuente principal de los planes de acción del sujeto y por lo tanto, pueden influir en su vida cotidiana. De esta manera los autores utilizan los términos prominencia o importancia relativa o self ideal y el de jerarquía de saliencia o self circunstancial. La prominencia es el equivalente a lo subjetivo, a la importancia de ese estado para el individuo, en cuanto a cómo él se imagina a sí mismo. La saliencia representa el self circunstancial; la jerarquía resultante de las identidades de rol en términos de su saliencia representa un orden relativo de prioridad frente a posibles actuaciones en una situación determinada. La situación de una identidad en esta jerarquía depende de la prominencia de la identidad, su necesidad de apoyo o legitimación, la necesidad o deseos de la persona de satisfacciones extrínsecas o intrínsecas obtenidas a través de su actuación y la oportunidad percibida. En consecuencia, para estos autores, la jerarquía de la prominencia se constituye en el más básico determinante de la saliencia.

Asimismo, aportan el concepto de relevancia de la identidad, el cual sostiene que una persona tiene múltiples identidades, tales como padre, esposo o un trabajador. Las mismas se organizan en una jerarquía de relevancia dentro de las cuales algunas identidades son más importantes y sobresalientes que otras (Habib \& Lancaster, 2010; Pasley, Kerpelman \& Guilbert, 2001). Es decir que esta relevancia de identidad se basa en el compromiso con el papel social. En otras palabras, esta conducta de rol refleja los diversos significados de quiénes son los padres. No hay una sola manera de ser padre, y por lo tanto hay variaciones en la manera de ejercer el rol (Rane \& McBride, 2000), lo que significa que el contenido del rol del padre puede depender de factores tales como la etapa del ciclo de vida, la cultura, las circunstancias sociales, el período histórico y las relaciones particulares o el contexto interpersonal en las que se produce la paternidad (Burgess, 1997; Colman, A. \& Colman, L., 1988; Lamb, 1997). Teóricamente, el contenido del estado de padre sugiere ciertas consecuencias, un hombre cuyo estado de jerarquía de importancia está dominada por la importancia relativa de sí mismo como un padre, que es el soporte emocional de la madre de su bebé, tiende a involucrarse con el cuidado y protección del bebé. Sin embargo, un hombre que se identifica más estrechamente con su propio padre como un proveedor tiende a pasar más horas en el trabajo en lugar de dedicar tiempo al cuidado de su hijo.

\section{TEORÍA SOCIAL DE LA IDENTIDAD DE EAGLY Y WOOD} (1999)

Esta teoría postula que las personas ocupan roles y que los mismos dependen de la elección de la persona, las presiones socioculturales biológicas, las cualidades psicológicas y a su vez los comportamientos relacionados con adaptarse a esas funciones. Por ejemplo, el papel biológico de la mujer en la maternidad (embarazo, parto y lactancia), junto con expectativas culturales de la misma, colocan a la mujer en un papel diferente al de los hombres que tienen una contribución biológica más pequeña. De esta manera, esta te- 
oría explica que las mujeres y los hombres pueden diferir psicológicamente antes del nacimiento del hijo debido a que ocupan diferentes roles en relación con el género (Palkovitz \& Palm, 2009).

Esto significa que la sociedad mantiene restricciones y oportunidades diferentes para los hombres y mujeres, que conducen a una división diferenciada del trabajo por género (Eagly \& Wood, 1999). Es decir que dicha fuerza de trabajo, diferenciadas por género, lleva a que hombres y mujeres se adapten psicológicamente a los roles marcados por la sociedad (Katz-Wise et al., 2010). De acuerdo con la teoría social estructural, hombres y mujeres sufren cambios psicológicos relacionados con el grado de alteración de sus roles sociales. Por lo tanto, de los padres que están haciendo la transición a la paternidad por primera vez, se espera que cambien más dramáticamente que los padres que tienen más de un hijo (Katz-Wise et al., 2010).

\section{DESARROLLO DE LA IDENTIDAD PATERNA DURANTE LA LLEGADA DE SU HIJO}

En relación con los cambios en la identidad del padre ante la llegada de su hijo, la paternidad puede ser vivida como una experiencia de entrega total por el hijo, es decir, como una experiencia de entrega gratificante $\mathrm{y}$ de autotrascendencia, o vivida como una pérdida de libertad asociada a sentimientos de tristeza e inadecuación. Según Fuller (2000) la paternidad como entrega total se manifiesta a través de la presencia disponible para entregar afecto y cuidado al hijo, como también de brindar las condiciones materiales necesarias para que el hijo se desarrolle.

Asimismo, los estudios concluyen que el hecho de ser padre implica el cierre de la etapa juvenil y el comienzo de la vida adulta (Fuller, 2000). De acuerdo con esta investigación, esto ocurre cuando hay efectos positivos en la identidad de los padres y adquieren una visión trascendente de la vida (Ohashi \& Asano, 2012; Swain, 2011). Esta implica adquirir mayor madurez, aumentar la autoestima y sentirse más acompañado.
Herzog y Lebovici (1995) enfatizan la relación de apoyo mutuo que debe existir en la pareja, señalando que uno de los procesos más importantes de la transición a la paternidad tiene que ver con la redefinición de la identidad de hombre y esposo hacia padre. Entre los procesos psicológicos que esta tarea implica, además de crear un lazo con el bebé, el padre también debe lograr la convicción interna de que puede cuidar de él. Los autores señalan que el padre puede ayudar a la madre en el proceso de transición a la parentalidad, pero él también necesita sostén para paternalizarse. Además de necesitar del apoyo afectivo de la madre para volverse un padre interesado, el proceso de paternalización estaría facilitado por la participación del padre en el deseo de tener un hijo, por la preparación para el parto y por su presencia en la sala de parto (Chen, Enright \& Tung, 2016; Kowlessar et al., 2014).

\section{LA INFLUENCIA DEL APEGO EN LA IDENTIDAD PATERNA}

La teoría del apego (Bowlby, 1980) explica que los seres humanos están dotados naturalmente con un sistema conductual de apego que motiva a la gente a relacionarse con otras personas importantes en su entorno. Estas relaciones con las figuras de apego contribuyen al desarrollo de modelos generalizados de sí mismo y los demás. Dichos modelos de trabajo abarcan una amplia gama de contenido cognitivo y afectivo, incluyendo recuerdos episódicos de las interacciones pasadas con las figuras de apego (Collins, Guichard, Ford \& Feeney, 2004).

Asimismo, se ha demostrado que las relaciones tempranas con las figuras de apego contribuyen al desarrollo de modelos generalizados, en donde dichos modelos influyen tanto a nivel cognitivo como afectivo, incluyendo los recuerdos de interacciones pasadas con las figuras de apego, las expectativas generales acerca de cómo las figuras de apego se comportarán en situaciones futuras, metas relacionadas con los planes y las manera de llevar a cabo dicha metas y planes (Collins et al., 2004; Habib \& Lancaster, 2010). 
Durante la transición a la paternidad, las propias experiencias de los nuevos padres con sus familias de origen pueden influir en los modelos o las representaciones mentales del funcionamiento de la familia a nivel consciente e inconsciente (Cohen \& FinziDottan, 2005; Habib \& Lancaster, 2010).

\section{TRANSMISIÓN INTERGENERACIONAL}

Palkovitz y Palm (2009) y Van Ijzendoorn (1992) describen a la transmisión intergeneracional de los estilos de crianza como la influencia de las generaciones anteriores en las actitudes y comportamientos de los nuevos padres. Así resultan modelos de crianza negativos tales como el rechazo a los hijos, maltrato infantil, entre otros (Weiss, Dodge, Bates \& Pettit, 1992) y positivos, por ejemplo afecto, apoyo, etc. (Simon \& Baxter, 1993).

Algunos estudios apoyan la hipótesis de que los padres son más propensos a estar involucrados si tuvieron un modelo de paternidad positivo en la infancia, mientras que otros apoyan la hipótesis de que la participación es mayor entre los padres que carecían de un modelo positivo, debido a que esta falta los llevaba a compensar la participación en las vidas de sus hijos (Parke, 2002; Van Ijzendoorn, 1992).

Según Espasa (2004) las identificaciones proyectivas de los padres con respecto al recién nacido llevan no sólo a las representaciones del pasado de los propios padres como niños sino, también, a representaciones de sus propios padres y de otras personas significativas de su infancia. Sin embargo, en este contexto de las relaciones padres - hijos, el término de identificación proyectiva no necesariamente tiene implicaciones patológicas (Palkovitz \& Palm, 2009). Por el contrario, en las identificaciones proyectivas de los padres es donde el niño a menudo desempeña un papel positivo en el desarrollo y estructuración psíquica de éste. Esto está estrechamente relacionado con el hecho de que la llegada de un bebé ofrece a los padres la oportunidad de revivir y / o corregir, en su relación con el bebé, ciertas ex- periencias y relaciones (Bouchard, 2012; Espasa, 2004).

Con respecto a los vínculos tempranos y con las parejas, se ha demostrado que la calidad del matrimonio dentro de la familia de origen se asocia con la calidad de matrimonios de la siguiente generación. Este impacto puede ser particularmente fuerte durante la transición a la paternidad (Cowan, C.P. \& Cowan, P.A., 2000).

Por ejemplo, las parejas que tuvieron conflictos con su familia de origen han demostrado una mayor disminución en la satisfacción marital desde el principio del embarazo hasta 18 meses después del parto (Cowan, C. P., Cowan, P.A., Heming \& Miller, 1991). Del mismo modo, Belsky e Isabella (1985) encontraron que cuando la persona recuerda haber sido criada en un hogar cálido y enriquecedor, evidencia descensos menores en la calidad de su relación romántica; Cox y colaboradores (1999) encontraron que las clasificaciones de apego adulto de padres moderaban la asociación entre la transición a la paternidad y la calidad de la relación romántica. Específicamente, los maridos clasificados con apego inseguro mostraron una disminución del afecto hacia sus mujeres durante la transición a la paternidad.

Estos hallazgos ponen de manifiesto cómo las experiencias con la familia de origen son relevantes e influyen en la calidad de la relación romántica después convertirse en padre. Esto significa que la transición a la paternidad evoca pensamientos acerca de las experiencias en la familia de origen y el tenor de esos recuerdos tiene injerencia sobre las familias (Bouchard, 2012; Perren, Von Wyl, Burgin, Simoni \& Von Klitzing, 2005).

\section{RELACIÓN DE PAREJA}

El período que rodea al nacimiento del primer hijo en la pareja, es a menudo estresante y puede tener efectos nocivos sobre las personas y las relaciones, debido a que las parejas deben hacer frente a grandes cambios de roles (Cowan et al.,1999). Es decir, que la llegada de un hijo puede ser un momento estre- 
sante para las madres y los padres debido a los grandes cambios que se generan en la vida de la transición de una díada familia a una tríada (Fish, Stifler \& Belsky, 1993).

De esta manera, con la llegada del primer hijo, las parejas deben reorganizar sus roles con respecto a la realización de tareas del cuidado de los niños, es decir que los mismos tienen que negociar cómo y quién pondrá en práctica diversas tareas específicas relacionadas con el cuidado del bebé (Castellano, Velotti, Crowell \& Zavattini, 2014).

Como ya se ha mencionado, la llegada de un hijo representa una crisis vital y evolutiva dentro del ámbito familiar, puesto que su nacimiento provoca un cambio en la estructura de la pareja, lo que lleva a modificar la manera de relacionarse y por ende, los padres deben atravesar un proceso de readaptación (Kluwer, 2010), en donde las demandas y las necesidades de su hijo, la atención, los gastos, entre otros, ocasionan niveles altos de ansiedad y estrés. Para Mortensen, Torsheim, Melkevik y Thuen (2012) es esperable que la llegada de un hijo refuerce las situaciones previas que caracterizaban la relación de pareja. Lo más amenazado suele ser la intimidad conyugal, que necesita ser resguardada.

Estos cambios que genera el nacimiento de un hijo pueden afianzar la unión de la pareja o afectar y romper su equilibrio al punto de llevarla incluso a la separación (Kowlessar et al., 2014; Lawrence, Rothman, Cobb \& Bradbury, 2012; Miller, 2011).

Esto significa que con el nacimiento del hijo la nueva familia deberá construir otra dinámica donde se asuman al mismo tiempo dos papeles: la pareja conyugal y la pareja parental. Los padres deben articular la función masculina o femenina con la paterna o materna y actuar esos diferentes roles según la situación. La función materna no debe anular la sensualidad femenina y el hombre debe ser el responsable de buscar su tiempo y espacio en la intimidad de la pareja (Lawrence et al., 2012).

Asimismo, se ha comprobado que durante este período la frecuencia de actividades matrimoniales, como pasar tiempo juntos y la actividad sexual tienden a disminuir (Cowan,
C.P. \& Cowan, P.A., 2000; Fillo et al., 2015). Las investigaciones sostienen que estos cambios llevan a la insatisfacción matrimonial. Shapiro, Gottman y Carrere (2000) sustentan que la transición a la paternidad es sobre todo exigente para las mujeres porque por lo general asumen la responsabilidad de ser los principales cuidadores del niño (MorenoRosset et al., 2016).

Los estudios realizados hasta el momento han identificado diversas variables que interfieren en la satisfacción de la pareja durante la transición a la paternidad (Kluwer, 2010; Moreno-Rosset et al., 2016). Las mismas son duración de la relación, la planificación del embarazo, el divorcio en la familia de origen, visiones religiosas, edad, nivel de educación e ingresos, entre otros (LachanceGrzela \& Bouchard, 2009).

En relación con lo anterior, Cowan, P.A. y Cowan, C.P. (1988) concluyeron que la transición a la paternidad incrementa las dificultades ya existentes entre las parejas. Los mismos argumentan que los comportamientos de las parejas se modifican durante el embarazo hasta meses o años después del nacimiento de un niño (Bradbury, Fincham \& Beach, 2000; Fillo et al., 2015).

Sin embargo, no todas las parejas reportan un descenso en la calidad del matrimonio a través de la transición a la paternidad, en algunos casos muestran mejoras (Belsky \& Rovine, 1990). Esto significa que los cambios en la calidad marital están asociados con la satisfacción de la pareja con respecto a la división de las tareas domésticas y el cuidado de los niños (Levy-Shiff, 1994), la personalidad y las variables demográficas, las características del niño y la salud mental de los padres (Adamsons, 2013; Castellano et al., 2014; Cox et al., 1999).

Por tanto, muchas investigaciones toman la satisfacción marital como un predictor más frecuente en la participación paterna. Las mismas apoyan la asociación positiva entre la satisfacción marital de padres primerizos o la calidad de su relación de pareja y la cantidad y calidad de la participación del padre en el cuidado de los niños (Lee \& Doherty, 2007). 
Además de las características personales, existen características que parecen influir sobre la vulnerabilidad durante la transición a la paternidad y en la relación de pareja. Estas características suelen incluir procesos sociales como la resolución de problemas, el nivel de compromiso en la relación romántica, la disposición de la pareja para tener un hijo, el nivel de confianza, entre otros (Ohashi \& Asano, 2012; Swain, 2011).

Finalmente, la relación matrimonial (romántica) establece el clima emocional de la familia (Cummings \& Davies, 2002) y tanto la relación conyugal como la relación entre padres e hijos están interrelacionadas (Gable, Belsky \& Cmic, 1992), lo que significa que la calidad de la relación entre los padres afecta el desarrollo del niño de tal manera que los problemas que haya entre los padres aumentan el riesgo de conducta y dificultades emocionales de niños y adolescentes (Cui, Conger \& Lorenz, 2005).

Se ha demostrado que los hombres que apoyan el embarazo de su pareja pueden formar un compromiso temprano con los roles de paternidad adquiriendo una identidad paterna saliente (Berlin, Cassidy \& Belsky, 1995). Por el contrario, los hombres que no apoyan o no están involucrados durante el embarazo no estarían motivados para asumir los roles de padre (Berlin et al., 1995; Habib \& Lancaster, 2010).

Por ello es necesario reconocer que la transición a la paternidad es un momento de estrés, así como de felicidad y mayor compromiso de la pareja, que da forma a las expectativas de un hombre y comportamientos acerca de su rol de padre (Fillo et al., 2015; Ihinger-Tallman \& Cooney, 2005).

\section{Discusión}

El estudio que se informa tuvo como objetivo realizar una revisión bibliográfica relacionada con la transición a la paternidad y estuvo centrado en explorar diversas teorías e investigaciones acerca también de la construcción de la identidad paterna durante la gestación y nacimiento de su hijo.
Los estudios citados demuestran que esta transición es un proceso multidimensional en términos de individuo, pareja, trabajo, familia de origen y factores de niño (Habib, 2012). Este artículo se ha centrado en el sentido de sí mismo del padre dentro del marco de la teoría de la identidad y la transición paterna, sintetizado y enlazando diversos enfoques que permiten comprender los sentimientos y conductas paternas durante ese período de transición.

A lo largo de esta revisión se ha observado que factores tales como la relación matrimonial, las demandas de trabajo, las relaciones tempranas con los padres y el género, entre otros, inciden en la construcción de la identidad paterna (Palkovitz \& Palm, 2009). Esto significa que la paternidad no es solo un hecho biológico sino que intervienen diversos factores en la construcción y configuración de la misma (Palkovitz \& Palm, 2009), ya que al igual que en la madre, en el padre también se presenta una crisis vital y evolutiva donde se ponen en juego el desarrollo de su identidad, su historia personal, su estructura de personalidad, la situación presente, las características del bebé y sus vínculos pasados y presentes (Nieri, 2015; Oiberman, 2008).

Conocer estos estudios permite entender y explicar los procesos psicoafectivos que transita un padre durante la construcción de su paternidad, haciendo hincapié en el significado y desarrollo del nuevo estatus y las consecuencias que tiene el mismo en el involucramiento de los nuevos padres con sus niños.

Además, las investigaciones citadas evidencian la necesidad de entender lo que le ocurre al padre durante su transición, ya que como la lleve adelante influirá en la relación con su pareja e hijo (Habib \& Lancaster, 2010; Moreno-Rosset et al., 2016).

Sin embargo, a lo largo de la búsqueda bibliográfica, la literatura e investigaciones sobre la participación paterna no han diferenciado y comparado sistemáticamente las dimensiones cualitativas e instrumentales de los roles e identidades paternas. Igualmente, no se encontraron investigaciones que analicen lo que le ocurre al padre durante el pro- 
ceso de gestación de su hijo y cómo reacciona en diversos momentos de este proceso. Finalmente, se han hallado pocas investigaciones que estudien empíricamente este tema.

Futuras líneas de investigación deberán estudiar las reacciones, emociones y conductas paternas durante el desarrollo y la transición hacia la paternidad.

Al ser un constructo poco estudiado, es necesario hacer estudios de tipo longitudinal con el fin de poder determinar: (a) ¿Cuándo comienza la transición?, (b) ¿Pueden reconocer los padres el inicio de la transición?, (c) ¿Cuáles son las características que se presentan durante su desarrollo? y (d) La imagen que el padre tenga de sí mismo ¿predice la participación paterna? (a nivel de compromiso, la accesibilidad y las responsabilidades del niño y el grado de vinculación con el niño).

\section{REFERENCIAS BIBLIOGRÁFICAS}

Adamsons, K. (2013). Predictors of relationship quality during the transition to parenthood. Journal of Reproductive and Infant Psychology, 31(2), 160-171. http://dx.doi.org/10. 1080/02646838.2013.791919

Arnal-Remón, B., Moreno-Rosset, C., RamírezUclés, I. \& Antequera-Jurado, R. (2015). Assessing depression, anxiety and couple psychological well-being in pregnancy: A preliminary study. Journal of Reproductive and Infant Psychology, 33(2), 128-139. http://dx.doi.org/10. 1080/02646838.2014.986648

Barclay, L., Donovan, J. \& Genovese, A. (1995). Men's experiences during their partner's first pregnancy: A grounded theory analysis. The Australian Journal of Advanced Nursing: A Quarterly Publication of the Royal Australian Nursing Federation, 13(3), 12-24.

Belsky, J. \& Isabella, R.A. (1985). Marital and parent-child relationships in family of origin and marital change following the birth of a baby: A retrospective analysis. Child Development, 56, 342-349.
Belsky, J. \& Kelly, J. (1994). Transition to parenthood. New York: Delacorte Press.

Belsky, J. \& Rovine, M. (1990). Patterns of marital change across the transition to parenthood: Pregnancy to three years postpartum. Journal of Marriage and the Family, 52, 5-19.

Berlin, L.J., Cassidy, J. \& Belsky, J. (1995). Infantmother attachment and loneliness in young children: A longitudinal study. Merrill-Palmer Quarterly, 41, 91-103.

Berman, P. \& Pedersen, A. (1987). Men's transition to parenthood: Longitudinal studies of early family experience. Hillsdale, NJ: Erlbaum.

Bibring, G.L. (1959). Some considerations of the psychological processes in pregnancy. Psychoanalytic Study of the Child, 14, 77-121.

Bouchard, G. (2012). Intergenerational transmission and transition to fatherhood: A mediatedmoderation model of paternal engagement. Journal of Family Psychology, 26(5), 747. http: //dx.doi.org/10.1037/a0029391

Bouchard, G. (2014). The quality of the parenting alliance during the transition to parenthood. Canadian Journal of Behavioural Science, 46, 20-28. http://dx.doi.org/10.1037/a0031259

Bowlby, J. (1980). Attachment and loss: Loss. New York, MY: Basic Books.

Bradbury, T.N., Fincham, F.D. \& Beach, S. (2000). Research on the nature and determinants of marital satisfaction: A decade in review. Journal of Marriage and the Family, 62, 964-980.

Buist, A., Morse, C.A. \& Durkin, S. (2003). Men's adjustment to fatherhood: Implications for obstetric health. Journal of Obstetric, Gynecological and Neonatal Nursing, 32(2), 172180.

Burgess, A. (1997). Fatherhood reclaimed: The making of the modern father. Londres: Vermilion.

Burke, P.J. \& Reitzes, D. (1991). An identity theory approach to commitment. Social Psychology Quarterly, 54, 239-251.

Cast, A.D. (2004). Well-being and the transition to parenthood: An identity theory approach. Sociological Perspectives, 47, 55-78. 
Castellano, R., Velotti, P., Crowell, J.A. \& Zavattini, G.C. (2014). The role of parents' attachment configurations at childbirth on marital satisfaction and conflict strategies. Journal of Child and Family Studies, 23(6), 1011-1026. http://dx.doi.org/10.1007/s10826-013-9757-7

Casullo, M. (2004). El nombre del hijo. Paternidad, maternidad y competencias simbólicas [The name of the son. Paternity, maternity and symbolic competencies]. Psicodebate. Psicología, Cultura y Sociedad, 5, 53-61. http://dx.doi.org/ 10.18682/pd.v5i0.451

Chen, E.Y.J., Enright, R.D. \& Tung, E.Y.L. (2016). The influence of family unions and parenthood transitions on self-development. Journal of Family Psychology, 30(3), 341. http://dx.doi. org/10.1037/fam0000154.

Cohen, O. \& Finzi-Dottan, R. (2005). Parent-child relationships during the divorce process; from attachment theory and intergenerational perspective. Contemporary Family Therapy, 27(1), 81-99.

Collins, N.L., Guichard, A.C., Ford, M.B. \& Feeney, B.C. (2004). Working models of attachment: New developments and emerging themes. En W.S. Rholes \& J.A. Simpson (Eds.), Adult attachment: Theory, research, and clinical implications (pp. 196-239). NY: Guilford.

Colman, A. \& Colman, L. (1988). The father: Mythology and changing roles. Wilmette, Illinois: Chiron.

Cowan, C.P. \& Cowan, P.A. (2000). When partners become parents: The big life change for couples. Mahwah, NJ: Erlbaum.

Cowan, C.P., Cowan, P.A., Heming, G. \& Miller, N.B. (1991). Becoming a family: Marriage, parenting, and child development. En P.A. Cowan \& E.M. Hetherington (Eds.), Family transition: Advances in family research (Vol. 2, pp. 79-109). Hillsdale, NJ: Erlbaum.

Cowan, P.A. \& Cowan, C.P. (1988). Changes in marriage during the transition to parenthood: Must we blame the baby? En G.Y. Michaels \& W.A. Goldberg (Eds.), The transition to parenthood: Current theory and research (pp. 114154). New York: Cambridge University Press.
Cox, M.J., Paley, B., Burchinal, M. \& Payne, C.C. (1999). Marital perceptions and interactions across the transition to parenthood. Journal of Marriage and the Family, 61, 611-625.

Cui, M., Conger, R.D. \& Lorenz, F.O. (2005). Predicting change in adolescent adjustment from change in marital problems. Developmental Psychology, 41, 812-823.

Cummings, E.M. \& Davies, P.T. (2002). Effects of marital conflict on children: Recent advances and emerging themes in process-oriented research. Journal of Child Psychology and Psychiatry, 43, 31-63.

de Montigny, P. \& de Montigny, F. (2013). Conceiving a first child: fathers' perceptions of contributing elements to their decision. Journal of Reproductive and Infant Psychology, 31, 274-284. http://dx.doi.org/10.1080/02646868. 2013.809519

Deave, T. \& Johnson, D. (2008). The transition to parenthood: What does it mean for fathers? Journal of Advanced Nursing, 63(6), 626-633. http://dx.doi.org/10.1111/j.1365-2648.2008.04 748.x

Draper, J. (2003). Men's passage to fatherhood: An analysis of the contemporary relevance of transition theory. Nursing Inquiry, 10(1), 66-78.

Durkheim, E. (1951). Suicide. Glencoe, IL: The Free Press.

Eagly, A.H. \& Wood, W. (1999). The origins of sex differences in human behavior: Evolved dispositions versus social roles. American Psychologist, 54, 408-423.

Ehrensaft, D. (1987). Parenting together: Men and women sharing the care of their children. New York: Free Press.

Espasa, F.P. (2004). Parent-infant psychotherapy, the transition to parenthood and parental narcissism: Implications for treatment. Journal of Child Psychotherapy, 30(2), 155-171.

Feeney, J.A., Hohaus, L., Noller, P. \& Alexander, R.P. (2001). Becoming parents. Cambridge: Cambridge University Press.

Fillo, J., Simpson, J.A., Rholes, W.S. \& Kohn, J.L. (2015). Dads doing diapers: Individual and relational outcomes associated with the division 
of child care across the transition to parenthood. Journal of Personality and Social Psychology, 108, 298-316. http://dx.doi.org/10.10 37/a0038572

Fish, M., Stifler, C. \& Belsky, J. (1993). Early patterns of mother-infant dyadic interactions: Infant, mother, and family demographic antecedents. Infant Behavior and Development, 16, 118.

Fuller, N. (2000). Significados y prácticas de paternidad entre varones urbanos del Perú [Meanings and parenting practices among urban males Perú]. Lima: PUCP.

Gable, S., Belsky, J. \& Cmic, K. (1992). Marriage, parenting, and child development: Progress and prospects. Journal of Family Psychology, 5, 276-294.

Gage, J. \& Kirk, R. (2002). First-time fathers: Perceptions of preparedness for fatherhood. Canadian Journal of Nursing Research, 34(4), 15-24.

Genesoni, L. \& Tallandini, M.A. (2009). Men's psychological transition to fatherhood: An analysis of the literature, 1989-2008. Birth, 36(4), 305-317.

Greenberg, M. \& Morris, N. (1974). Engrossment: The newborn's impact upon the father. American Journal of Orthopsychiatry, 44(4), 520531.

Habib, C. (2012). The transition to fatherhood: A literature review exploring paternal involvement with identity theory. Journal of Family Studies, 18(2-3), 103-120. http://dx.doi.org/10. 5172/jfs.2012.18.2-3.103

Habib, C. \& Lancaster, S. (2010). Changes in identity and paternal-foetal attachment across a first pregnancy. Journal of Reproductive and Infant Psychology, 28(2), 128-142.

Herzog, J.M. \& Lebovici, S. (1995). El padre. En S. Lebovici \& F. Weil-Halpern (Eds.), La psicopatología del bebé [The baby psychopathology], (pp. 70-78). México, DF: Siglo XXI.

Hill, R. (1949). Families under stress. NY: Harper.

Höfner, C., Schadler, C. \& Richter, R. (2011).

When men become fathers: Men's identity at the transition to parenthood. Journal of Comparative Family Studies, 42, 669-686.

Ihinger-Tallman, M. \& Cooney, T.M. (2005). Families in context. Los Angeles: Roxbury Publishing.

Ihinger-Tallman, M., Pasley, K. \& Buehler, C. (1993). Developing a middle-range theory of father involvement postdivorce. Journal of Family Issues, 14(4), 550-571.

Katz-Wise, S.L., Priess, H.A. \& Hyde, J.S. (2010). Gender-role attitudes and behavior across the transition to parenthood. Developmental Psychology, 46(1), 18. http://dx.doi.org/10.1037/a 0017820.

Keizer, R., Dykstra, P.A. \& Poortman, A.R. (2010). The transition to parenthood and wellbeing: The impact of partner status and work hour transitions. Journal of Family Psychology, 24(4), 429. http://dx.doi.org/10.1037/a0 020414

Kluwer, E. (2010). From partnership to parenthood: A review of marital change across the transition to parenthood. Journal of Family Theory \& Review, 2(2), 105-125.

Kowlessar, O., Fox, J.R. \& Wittkowski, A. (2014). The pregnant male: A metasynthesis of firsttime fathers' experiences of pregnancy. Journal of Reproductive and Infant Psychology, 33(2), 106-127. http://dx.doi.org/10.1080/02646838. 2014.970153

Kuhn, M.H. (1960). Self attitudes by age, sex, and professional training. Sociological Quarterly, 1(1), 39-55.

Lachance-Grzela, M. \& Bouchard, G. (2009). The well-being of cohabiting and married couples during pregnancy: Does pregnancy planning matter? Journal of Social and Personal Relationships, 26(2), 607-627.

Lamb, M.E. (1997). The development of fatherinfant relationships. En M.E. Lamb (Ed.), The role of the father in child development. New York: Whiley.

Lawrence, E., Rothman, A.D., Cobb, R.J. \& Bradbury, T.N. (2012). Marital satisfaction across the transition to parenthood: Three eras of research. Washington, DC: APA. 
Lee, C. \& Doherty, W. (2007). Marital satisfaction and father involvement during the transition to parenthood. Fathering, 5(2), 75-96.

LeMasters, E.E. (1957). Parenthood as crisis. Marriage and Family Living, 19, 352-355.

Levy-Shiff, R. (1994). Individual and contextual correlates of marital change across the transition to parenthood. Developmental Psychology, 30, 591-601.

Lupton, D. \& Barclay, L. (1997). Constructing fatherhood: Discourses and experiences. Londres: Sage.

May, K.A. (1982). Factors contributing to first-time fathers' readiness for fatherhood: An exploratory study. Family Relations, 31, 353-361.

McCall, G.J. \& Simmons, J.L. (1978). Identities and interactions: An examination of human associations in everyday life. New York: The Free.

Miller, T. (2011). Falling back into gender? Men's narratives and practices around first-time fatherhood. Sociology, 45(6). http://dx.doi.org/ 10.1177/0038038511419180

Moreno-Rosset, C., Arnal-Remón, B., AntequeraJurado, R., \& Ramírez-Uclés, I. (2016). Anxiety and psychological wellbeing in couples in transition to parenthood. Clínica y Salud, 27(1), 29-35. http://dx.doi.org/10.1016/j.c lysa.2016. 01.0041130-5274

Mortensen, O., Torsheim, T., Melkevik, O. \& Thuen, F. (2012). Adding a baby to the equation. Married and cohabiting women's relationship satisfaction in the transition to parenthood. Family Process, 51, 122-139. http://dx.doi.org /10.1111/j.1545-5300.2012.01384.x

Nieri, L. (2015). Construcción y validación del Cuestionario de Sensibilidad Paterna [Construction and validation of the Paternal Sensitivity Questionnaire]. Psicodebate, 15(2), 55-82.

Nyström, K. \& Öhrling, K. (2004). Parenthood experiences during the child's first year: Literature review. Journal of Advanced Nursing, 46(3), 319-330.

Ohashi, Y. \& Asano, M. (2012). Transition to early parenthood, and family functioning relationships in Japan: A longitudinal study. Nursing and Health Sciences, 14, 140-147. http://dx. doi.org/10.1111/j.1442-2018.2011.00669.x

Oiberman, A. (2008). Observando a los bebés: Técnicas vinculares madre-bebé y padre-bebé [Noting babies: Relational techniques motherbaby and father-baby]. Buenos Aires: Lugar.

Palkovitz, R. \& Palm, G. (2009). Transitions within fathering. Fathering, 7(1), 3.

Parke, R.D. (2002). Fathers and families. En M.H. Bornstein (Ed.), Handbook of parenting: Volume 3 (pp. 27-73). Mahwah, NJ: Erlbaum.

Pasley, K., Kerpelman, J. \& Guilbert, D.E. (2001). Gendered conflict, identity disruption, and marital instability: Expanding Gottman's model. Journal of Social and Personal Relationships, 18, 5-27.

Perren, S., Von Wyl, A., Burgin, D., Simoni, H. \& Von Klitzing, K. (2005). Intergenerational transmission of marital quality across the transition to parenthood. Family Process, 44, 441-459.

Pleck, J.H. (1997). Paternal involvement: Levels, sources, and consequences. En M.E. Lamb (Ed.), The role of the father in child development. New York: Wiley.

Price, S., McKenry, P. \& Murphy, M. (2000). Families across time: A life course. California. Roxbury: Publishing Company.

Rane, T.R. \& McBride, B.A. (2000). Identity theory as a guide to understanding fathers' involvement with their children. Journal of Family Issues, 21(3), 347-366.

Reynolds, J., Houlston, C. \& Coleman, L. (2014). Understanding relationship quality. London: OnePlusOne. Recuperado el 24 de agosto de 2016 de http://www.oneplusone.org.uk/wpcontent/uploads/2014/02/Understanding-Relation ship-Quality.

Rholes, W.S., Simpson, J.A., Kohn, J.L., Wilson, C.L., Martin, A.M., III, Tran, S. et al. (2011). Attachment orientations and depression: A longitudinal study of new parents. Journal of Personality and Social Psychology, 100, 567586.

Rossi, A.S. (1968). Transition to parenthood. Journal of Marriage and Family, 30, 26-39. 
Schumacher, K.L. \& Meleis, A.I. (1994). Transitions: A central concept in nursing. Image: Journal of Nursing Scholarship, 26, 119-127.

Shapiro, A., Gottman, J. \& Carrere, S. (2000). The baby and the marriage: Identifying factors that buffer against decline in marital satisfaction after the first baby arrives. Journal of Family Psychology, 14, 59-70.

Simon, E.P. \& Baxter, L.A. (1993). Attachment style differences in relationship maintenance strategies. Western Journal of Communication, 57, 416-430.

Strauss, R. \& Goldberg, W.A. (1999). Self and possible selves during the transition to fatherhood. Journal of Family Psychology, 13(2), 244-259.
Swain, J.E. (2011). Becoming a Parent - Biobehavioral and Brain Science Perspectives. Current Problems in Pediatric and Adolescent Health Care, 41, 192-196. http://dx.doi.org/10.1016/j. cppeds.2011.02.004

Testa, R. (2010). She wants, he wants: Couples's child bearing desires in Austria (Working Paper 3/2010). Vienna Institute of Demography.

Van Ijzendoorn, M.H. (1992). Intergenerational transmission of parenting: A review of studies in non-clinical population. Developmental Review, 12, 76-99.

Weiss, B., Dodge, K. A., Bates, J. E. \& Pettit, G.S. (1992). Some consequences of early harsh discipline, child aggression and maladaptive social information processing style. Child Development, 63, 1321-1335.

Consejo Nacional de Investigaciones

Cientificas y Técnicas (CONICET)

Fundación Universidad Argentina de la Empresa (UADE)

Ciudad Autónoma de Buenos Aires - República Argentina

Fecha de recepción: 24 de junio de 2016

Fecha de aceptación: 2 de marzo de 2017 